\title{
review
}

\section{Hideand run}

\section{Arginine-based endoplasmic-reticulum-sorting motifs in the assembly of heteromultimeric membrane proteins}

\author{
Kai Michdsen*, HebaoYuan* \& BlandheSdmappadh ${ }^{+}$ \\ Zentrum für M olekulare Biologie der Universität H eidelberg (ZM BH ), H eidel berg, Germany
}

\begin{abstract}
Arginine-based endoplasmic reticulum (ER)-localization signals are sorting motifs that are involved in the biosynthetic transport of multimeric membrane proteins. After their discovery in the invariant chain of the major histocompatibility complex class II, several hallmarks of these signals have emerged. They occur in polytopic membrane proteins that are subunits of membrane protein complexes; the presence of the signal maintains improperly assembled subunits in the ER by retention or retrieval until it is masked as a result of heteromultimeric assembly. A distinct consensus sequence and their position independence with respect to the distal termini of the protein distinguish them from other ER-sorting motifs. Recognition by the coatomer (COPI) vesicle coat explains ER retrieval. 0 ften, di-leucine endocytic signals occur close to arginine-based signals. Recruitment of 14-3-3 family or PDZ-domain proteins can counteract ER-localization activity, as can phosphorylation. This, and the occurrence of argininebased signals in alternatively spliced regions, implicates them in the regulated surface expression of multimeric membrane proteins in addition to their function in quality control.

Keywords: peptide sorting motifs; transport signals; ion channels;

quality control; CO PI coat

EMBO reports(2005) 6,717-722. doi:10.1038/sj.embor.7400480
\end{abstract}

\section{Introduction}

Subcellular compartments and the regulation of traffic between them allow the cell to separate important events. For example, in the secretory pathway, quality control of newly synthesized proteins occurs before downstream processing reactions take place. At the same time, transport machinery proteins, such as p24 proteins and SNAREs, and modifying enzymes need to be maintained in their compartments, as do resident proteins of the endoplasmic reticulum (ER). This is partly aided by the linear

\footnotetext{
Zentrum für M olekulareBiologieder Universität H eidelberg (ZM BH),

Im Neuenheimer Feld 282, D-69120 Heidelberg, Germany

*These authors contributed equally to this work

+Corresponding author. Tel: +49 (0) 622154 6898; Fax: +49 (0) 622154 5894;

E-mail: b.schwappach@zmbh.uni-heidelberg.de
}

Submitted 6 May 2005; accepted 17 June 2005 signals $-K D E L$ and $-K(X) K X X$, which represent well-characterized ER-localization signals for lumenal and membrane proteins, respectively (Teasdale \& Jackson, 1996). Here, we review a class of less well-characterized ER-sorting motifs: arginine (Arg)-based ER-localization signals. These signals were discovered in the context of major histocompatibility complex $(\mathrm{MHC}$ ) class II transport (Bakke \& D obberstein, 1990; Lotteau et al, 1990), and after this discovery, a seminal study investigated the determinants that localize one form of the invariant chain li (Fig 1A) to the ER (Schutze et al, 1994). This paper is entitled 'An N-terminal double-arginine motif maintains type II membrane proteins in the ER', which led to the general assumption that these motifs were specific for type II membrane proteins. In addition, one particular generalization of the data in this study initially biased our concept of these sorting signals: the authors stressed the spacing requirement of the arginine residues with respect to the proximal amino-terminus. Although this is consistent with the data presented, the spacing issue may have been overemphasized owing to the influence of the previously characterized carboxyterminal $-K(X) K X X$ signal that depends crucially on exact spacing (Nilsson et al, 1989). Accordingly, it took the field some time to realize that these sorting motifs are much more widespread than previously believed and can, in fact, be active at various positions of diverse polytopic membrane proteins (Fig 1, supplementary Table I online).

\section{Coupling the assembly state to forward transport}

Arg-based signals can localize a broad range of reporter proteins to the ER but their function has been documented mainly in membrane protein complexes that are destined to leave the ER, for example, in ATP-sensitive potassium $\left(\mathrm{K}_{\mathrm{ATP}}\right)$ channels (Fig 1C,D). These channels link the metabolic state of cells to their electrical excitability. The proper hetero-octameric assembly of four inward rectifier potassium (Kir) channel subunits and four sulphonylurea receptor (SUR) proteins is a prerequisite for $\mathrm{K}_{\text {ATP }}$ channels to exert their regulatory function in neurons or pancreatic $\beta$-cells. Each subunit contains one Arg-based signal that localizes unassembled subunits or incompletely assembled complexes to the ER. During heteromultimeric assembly, this 

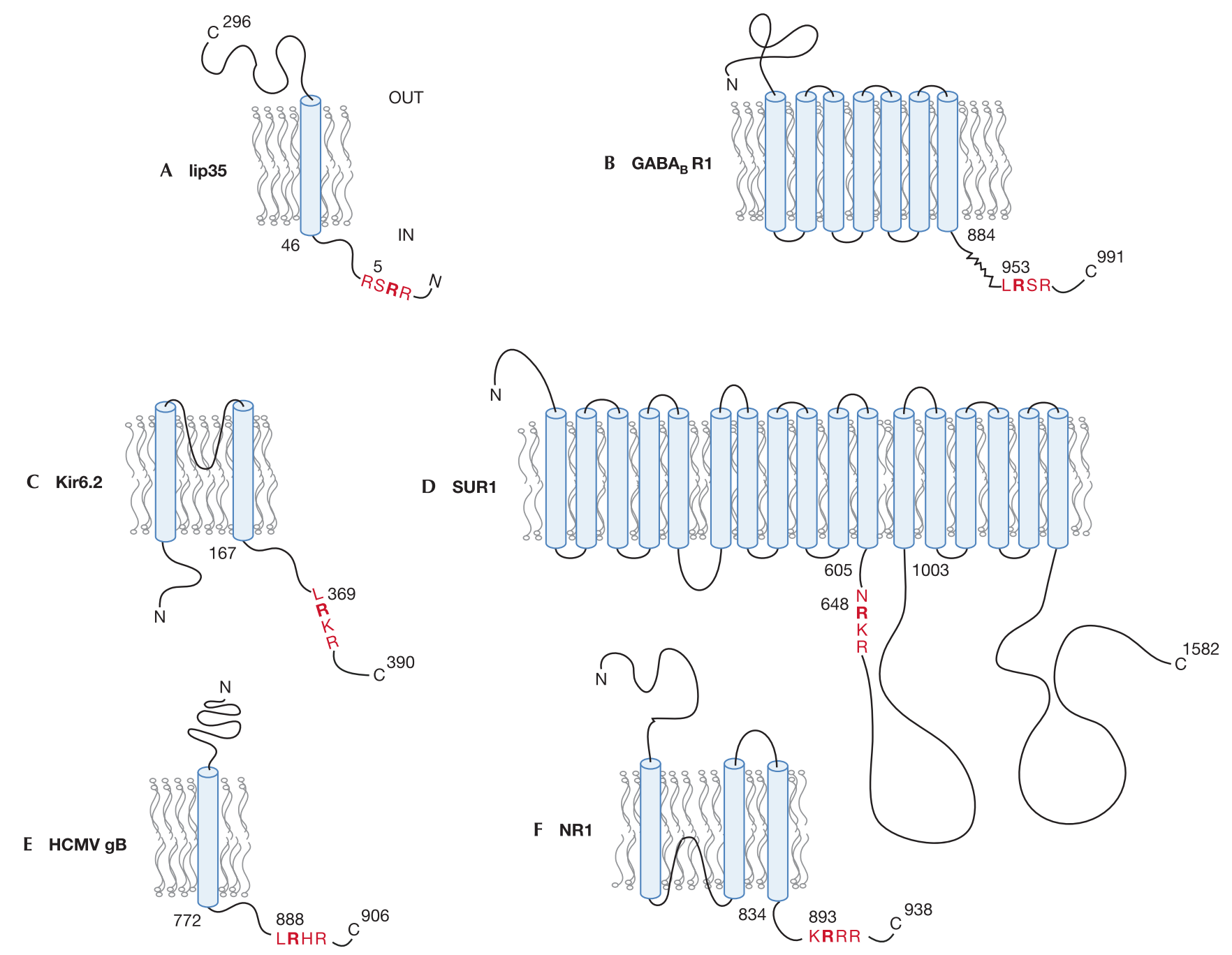

Fig1|Arginine-based signals in proteins of different topology. Numbers indicatethesize of cytosolic domains and the first Arg residue of theArg-based signal. Arg-based signals are indicated in red. (A) Invariant chain li p35 of major histocompatibility complex (M HC) class II (p35 is one of two variants owing to the use of alternativeinitiator methionines). (B) $\gamma$-aminobutyric acid $\left(\mathrm{GABA}_{\mathrm{B}}\right)$ receptor subunit 1. Thezigzag lineindicates a coiled-coil forming domain. (C) Kir6.2 is the pore-forming subunit of the $K_{\text {ATP }}$ channel. (D) SUR1 is the regulatory subunit of the $\mathrm{K}_{\text {ATP }}$ channel. (E) Glycoprotein B from human cytomegalovirus (HCM V). (F) N-methyl D-aspartate(N M DA) receptor subunit NR1-1.

signal is masked, which allows transport of the complex to the cell surface (Fig 2; Zerangue et al, 1999). Similarly, heterodimeric assembly of the $G$-protein-coupled $\gamma$-aminobutyric acid $\left(G_{B B A}\right)$ neurotransmitter receptor (Figs $1 B, 3 A$ ) is coupled to cell-surface transport of the complex by the presence of an Arg-based signal in one subunit (Margeta-Mitrovic et al, 2000). On the basis of these and additional examples (see below), it has become clear that an exposed Arg-based signal results in ER localization of the respective subunit or partial complex. In several cases, a di-leucine endocytic or lysosomal targeting motif also operates in the vicinity of the Arg-based signal ( $\mathrm{Hu}$ et al, 2003; Khalil et al, 2003; Margeta-Mitrovic et al, 2000; Ren et al, 2003a). This indicates a possible second mechanism for controlling the composition and number of membrane protein complexes at the cell surface.
The rediscovery of Arg-based signals in polytopic membrane proteins has spurred numerous investigations of putative signals in other membrane protein complexes. Most ion channels and neurotransmitter receptors can form alternative heteromultimers; the resulting combinatorial variety is the basis for the different electrical properties of neurons and for changes in synaptic strength. Interestingly, a functional Arg-based signal has been identified in the alternatively spliced C-terminal $\mathrm{Cl}$ cassette of the ionotropic $\mathrm{N}$-methyl D-aspartate (N M DA) glutamate receptor subunits N R1-1 and NR1-3 (Fig 1F; Scott et al, 2001; Standley et al, 2000; Xia et al, 2001), and subsequently in other ionotropic and metabotropic glutamate receptors (Chan et al, 2001; Ren et al, 2003a, 2003b). These recent findings suggest a role for Arg-based signals in the regulation of receptor complex assembly and movement beyond constitutive quality control as observed for the biosynthetic transport of $\mathrm{K}_{\text {ATP }}$ channels. 


\section{Consensus, position and distance from the membrane}

Our information on an Arg-based motif consensus sequence is based on naturally occurring signals (supplementary Table I online) and variants created by site-directed mutagenesis. In addition, a combinatorial screening approach was used to select active or inactive variants from a random library containing Kir6.2-derived -TLASXXRXRXXSLS C-terminal tails (Zerangue et al, 2001). The integration of this information led to the conclusion that Arg-based signals conform to the consensus $\Phi / \Psi / R-R-X-R$, in which $\Phi / \Psi$ denotes an aromatic or bulky hydrophobic residue and $X$ represents any amino acid. Generally, Iysine residues cannot substitute for arginine residues. More than two arginine residues gives rise to particularly strong sorting motifs, whereas the residue that precedes $R X R$ and the identity of $X$ itself can modulate the signal to an intermediate efficacy that results in significant steady-state Golgi localization. Indeed, quantitative trans-Golgi network processing and surface expression assays revealed that the variants identified from the random library cover a whole spectrum of differentially efficacious sorting signals (Zerangue et al, 2001). Negatively charged residues or small, non-polar side chains in these two positions usually render the signal inactive.

In contrast to the C-terminal $-K(X) K X X$ ER-localization signal, Arg-based signals do not need to be exposed at the distal terminus of a membrane protein in order to be functional. Consistently, naturally occurring signals are present in different cytosolic domains of polytopic membrane proteins (Fig 1; supplementary Table I online). Transplantation of the signal to various positions in a range of reporter proteins has convincingly shown that the motif behaves as a peptide transport signal with no other requirements but sufficient exposure. Accordingly, Arg-based signals present in different members of a protein family (for example, Kir6.1/2 or SUR1/2 KATP channel subunits) can reside in particularly poorly conserved regions of the proteins. Accessibility of the signal depends not only on its position in the membrane protein but also on its proximity to a transmembrane segment. Careful comparison of the efficacy of an Arg-based and a -KKXX ER-localization signal (Shikano \& Li, 2003) revealed that Arg-based signals are functional in a zone approximately 16-46 $\AA$ away from the lipid bilayer, whereas the - KKXX signal was more active when positioned closer to the cytoplasmic side of the membrane leaflet (Shikano $\&$ Li, 2003). The different spacing requirements of the two ER-localization signals with respect to their position in the protein and to the preceding transmembrane segment strongly suggest that they are recognized by different receptor proteins.

\section{How and where are Arg-based signals recognized?}

The cellular machinery required to decode Arg-based signals is poorly characterized (Nufer \& Hauri, 2003). The coat protein complex I (CO PI) complex mediates the retrieval of -K(X)KXX-bearing membrane proteins (McMahon \& Mills, 2004), and so its role in the recognition of Arg-based signals has been tested ( $O$ 'Kelly et al, 2002; Yuan et al, 2003; Zerangue et al, 2001). The results support a role for $\mathrm{CO} \mathrm{PI}$ in the retrieval of membrane proteins that expose an Arg-based signal; however, the details of recognition, such as which subunit of the coat complex acts as the direct receptor for the sorting signal, are obscure. Recent experiments have shown that variants of the $-K(X) K X X$ signal are recognized by WD40

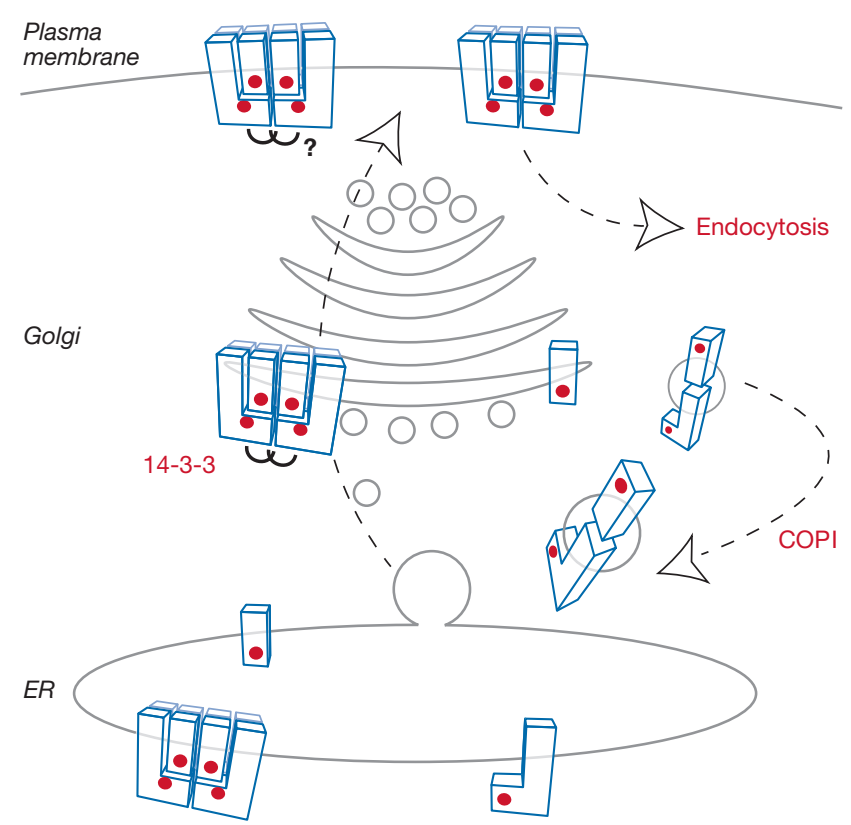

Fig2| M odel of $K_{\text {ATP }}$ channel transport. Larger and smaller rectangular shapes represent the SUR1 and Kir6.2 subunits, respectively. Arginine (Arg)based signals are shown as red dots. 14-3-3 proteins are depicted as joined brackets. These proteins have been implicated in the forward transport of $K_{\text {ATP }}$ channels (Yuan \&al, 2003), but their exact roleis unclear (Nufer \& Hauri, 2003). A di-leucine endocytosis signal in the vicinity of the Arg-based signal has been shown to mediate dynamin-dependent endocytosis ( $\mathrm{Hu}$ etal, 2003). COPI, coat protein complex I; ER, endoplasmic reticulum.

domains of either the $\alpha$ or the $\beta^{\prime}$ CO PI subunit (Eugster et al, 2004). The characteristic differences between $-\mathrm{K}(\mathrm{X}) \mathrm{KXX}$ and the Arg-based ER-localization signals discussed earlier strongly suggest that these peptide motifs are recognized by different binding pockets of the CO PI complex.

Furthermore, the mechanistic issue of whether Arg-based signals result in ER retention versus ER retrieval is intimately linked to the cellular machinery involved in the process. Recognition by COPI is consistent with the finding that Arg-based signals can mediate the retrieval of proteins to the ER as assessed by glycan analysis (Hardt et al, 2003). However, in many cases, only a minority of membrane proteins exposing an Arg-based signal receives any modification that would indicate ER exit. Therefore, true retention mechanisms might exist in addition to CO PI-mediated retrieval (N ufer \& Hauri, 2003).

\section{Masking of Arg-based signals}

The ability of the Arg-based sorting motif to act as a transient ER-localization signal is one of its most striking and consistent features. This raises the question of how the signal is inactivated on proper heteromultimeric assembly (Fig 3). Steric masking by a partner subunit is the simplest conceivable mechanism and has previously been proposed for the masking of a -KKXX signal in a heteromultimeric complex (Letourneur et al, 1995). $\mathrm{GABA}_{B}$ receptors illustrate this mechanism particularly well (Fig $3 A$ ) as the coiled-coil forming domains present in the $\mathrm{C}$-termini of both 


\section{A GABA $_{B} R$}

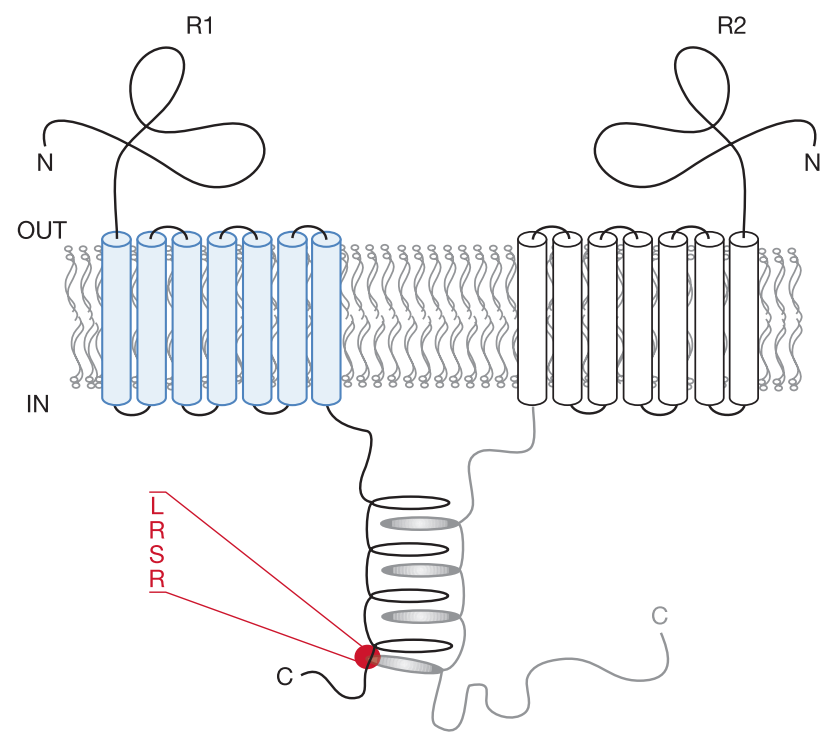

B MHC II

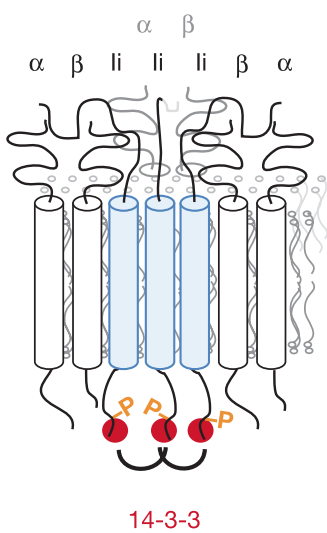

C NMDA R

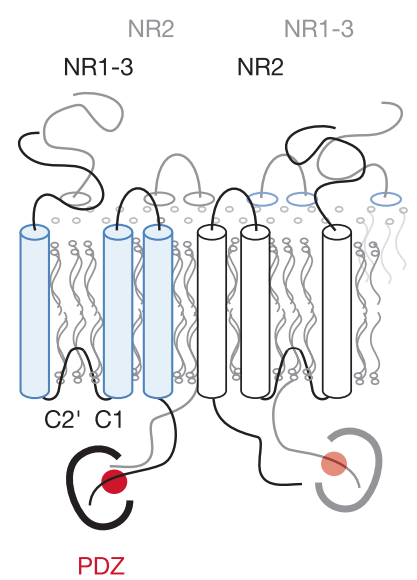

Fig3 M echanisms for the masking of arginine-based signals. Red dots symbolizetheArg-based signals. (A) Steric masking: coiled-coil forming domains in the two $\gamma$-aminobutyric acid $\left(G_{A B A}\right)$ receptor subunits hidethesignal present in $G_{A B A}$ R1-subunit (M argeta-M itrovic etal, 2000). (B) Phosphorylation of a residue in the vicinity of theArg-based signal in the major histocompatibility complex ( $\mathrm{M} \mathrm{HC}$ ) invariant chain recruits 14-3-3 proteins (joined brackets), which leads to signal shielding (Kuwana etal, 1998). Heteromultimeric assembly with M HC class II $\beta$-subunit also leads to steric masking (Khali \& al, 2003). H ow the two mechanisms relateto each other is unclear. (C) M asking by a PDZ-domain protein (large bracket) binding to the distal C-terminus of N-methyl D-aspartate receptor subunit NR1-3 (Standley etal, 2000).

$\mathrm{GABA}_{B}$ subunits have been shown to mediate masking, not only in the context of the full-length receptor, but also when transplanted to a reporter protein (Margeta-M itrovic et al, 2000). $\mathrm{K}_{\text {ATP }}$ channels (Fig 2) are another example of steric masking of the Arg-based signal after proper assembly. However, for these channels, the situation is more complex as 14-3-3 family proteins have been shown to recognize the C-terminal tail of one subunit, Kir6.2, provided it contains an Arg-based signal (Yuan et al, 2003). The hypothesis that 14-3-3 proteins can proofread the assembly state of multimeric proteins and promote the forward transport of properly assembled complexes by shielding the Arg-based signals needs to be tested in the context of the entire $\mathrm{K}_{\text {ATP }}$ channel complex (N ufer \& Hauri, 2003). Interestingly, this situation is reminiscent of the invariant chain Ii (Fig 3B): the MHC class II $\beta$-chain cytoplasmic tail was shown to overcome the Arg-based signal during heteromultimeric assembly of the Ii-M HC complex (Khalil et al, 2003). In addition, invariant chain phosphorylation and subsequent 14-3-3 recruitment were required for the forward transport of Ii (Kuwana et al, 1998). The example of the NMDA receptor splice variants NR1-1 or NR1-3 (containing the C-terminal C1 as well as the C2' cassette with a C-terminal PDZ-domain-interacting motif) provides a fascinating new perspective on the inactivation of the Arg-based signal: the phosphorylation of nearby residues has been proposed to inactivate the signal in the NMDA receptor subunit NR1-1 (Scott et al, 2001). Although the mechanism has been plausibly delineated, the ample use of negatively charged side-chains to introduce phospho-mimicking mutations close to the Arg-based signal is a source of concern: these changes might affect signal efficacy but do not show that such modulation happens as a consequence of an in vivo phosphorylation event (Scott et al, 2001, 2003; Standley et al, 2000; Xia et al, 2001). Finally, the Arg-based signal present in the NR1-3 subunit (Fig 3C) was shown to be masked by the recruitment of a PDZ-domain protein to the distal C-terminus of the protein (Scott et al, 2001; Standley et al, 2000; Xia et al, 2001). The issue of how Arg-based signals are masked is intimately connected to the question of where in the secretory pathway the crucial events occur (N ufer $\&$ Hauri, 2003) and to what extent early interactions impinge on later transport steps.

\section{0 ther functions?}

Strong Arg-based signals have been found in the C-termini of type I viral membrane proteins (Fig $1 \mathrm{E}$ ), the glycoprotein $B$ homologues of Epstein-Barr virus, herpes simplex virus type I and human cytomegalovirus (Lee, 1999; M eyer et al, 2002; M eyer \& Radsak, 2000). These viruses undergo a characteristic nuclear phase of maturation. Accordingly, studies have focused on the surprising finding that Arg-based signals are able to mediate access of viral membrane proteins to the inner nuclear membrane (Meyer et al, 2002; Meyer \& Radsak, 2000). This is in contrast to membrane proteins that are destined for the secretory pathway or ER-resident proteins that are normally excluded from this domain.

Arg-based signals can result in the steady-state localization of various reporter proteins to the ER. However, there is virtually no evidence that these signals do actually localize ER-resident proteins to this compartment. The $\mathrm{N}$-terminus of the ER resident glucosidase I contains a functional Arg-based signal that is, however, not required to localize the full-length protein (Hardt et al, 2003). 
Another plausible function of Arg-based signals might be the coupling of folding status to the forward transport of a membrane protein. This scenario, in which the signal is exposed due to improper folding, has been investigated for the cystic fibrosis transmembrane conductance regulator (CFTR) protein (Chang et al, 1999). The effect of replacing the arginine residues in four different putative Arg-based signals was impressive: the manipulation resulted in the plasma membrane targeting of the $\Delta$ F508 mutant form of the protein, which is normally degraded due to ER quality control and which is the cause of disease in most cystic fibrosis patients. However, one of the Arg-based signals that were manipulated is not present in mouse CFTR and another one occurs in a region previously shown to be sensitive to second-site suppressor mutations. Furthermore, the crucial defect in the $\Delta \mathrm{F} 508$ mutant protein has been shown recently to lie in the loss of a functional ER-exit signal rather than the exposure of normally masked ER-localization signals (Wang et al, 2004). Whether or not the transient exposure of Arg-based signals during folding mediates ER localization beyond the action of ER chaperones remains an important and technically challenging question. Evidence in favour of this concept has been provided by the exposure of active Arg-based signals as a consequence of truncating proteins (Hermosilla \& Schulein, 2001; Kupershmidt et al, 2002). O f particular interest is the interplay between such normally hidden signals and disease-causing mutations. This relationship has been investigated for SUR1 mutations that correlate with hyperinsulinism (Cartier et al, 2001) and vasopressin receptor 2 mutations that correlate with diabetes insipidus (Hermosilla et al, 2004). These experiments suggest that the degree of misfolding of a mutant protein determines whether or not Arg-based signals present in the protein impinge on its transport; for example, whether or not inactivation of an Arg-based signal allows forward transport of the mutant membrane protein.

\section{Conclusions and perspectives}

Arg-based signals are distinct from other known ER-localization signals with respect to their consensus sequence, positioning and function. In the future, we hope to understand the details of how the COPI coat recognizes the signal and results in the retrieval of membrane proteins. Additional cellular machinery may participate in mediating the ER localization of membrane proteins that contain an Arg-based signal. Steric masking is one main mechanism that leads to the inactivation of these signals in the course of heteromultimeric assembly, but it will be exciting to pursue further the role of phosphorylation, as well as the recruitment of additional proteins such as 14-3-3 in the masking of Arg-based signals. Eventually, the goal is to understand the physiological significance of Arg-based signal exposure and masking.

Supplementary information is available at EMBO reports online (http://www.emboreports.org).

\section{ACKN O W LED GEMENTS}

Wethank many colleagues from the Zentrum für M olekulare Biologie der Universität Heidel berg (ZM BH) and the Biochemistry Centre (BZH), as well as members of the Schwappach laboratory for critical reading of the manuscript. Our work is funded by the Deutsche Forschungsgemeinschaft (SFB638), the Landesstiftung Baden-W ürttemberg and the EM BO Young Investigator Programme.

\section{REFEREN CES}

Bakke O, D obberstein B (1990) M H C class II-associated invariant chain contains a sorting signal for endosomal compartments. Cell 63: 707-716

Cartier EA, Conti LR, Vandenberg CA, Shyng SL (2001) D efective trafficking and function of $K_{\text {ATP }}$ channels caused by a sulfonylurea receptor 1 mutation associated with persistent hyperinsulinemic hypoglycemia of infancy. Proc N atl Acad Sci U SA 98: 2882-2887

Chan WY, Soloviev M M, Ciruela F, M cllhinney RA (2001) M olecular determinants of metabotropic glutamate receptor 1B trafficking. Mol Cell Neurosci 17: 577-588

Chang XB, Cui L, H ou YX, Jensen TJ, Aleksandrov AA, M engos A, Riordan JR (1999) Removal of multiple arginine-framed trafficking signals overcomes misprocessing of $\Delta$ F508 CFTR present in most patients with cystic fibrosis. Mol Cell 4: 137-142

Eugster A, Frigerio G, Dale M, Duden R (2004) The $\alpha$ - and $\beta^{\prime}$-CO P W D 40 domains mediate cargo-selective interactions with distinct di-lysine motifs. Mol Biol Cell 15: 1011-1023

Hardt B, Kalz-Fuller B, A paricio R, Volker C, Bause E (2003) (Arg)3 within the $\mathrm{N}$-terminal domain of glucosidase I contains ER targeting information but is not required absolutely for ER localization. Glycobiology 13: $159-168$

Hermosilla R, Schulein R (2001) Sorting functions of the individual cytoplasmic domains of the $G$ protein-coupled vasopressin $V(2)$ receptor in Madin Darby canine kidney epithelial cells. M ol Pharmacol 60: 1031-1039

Hermosilla R, O ueslati M, D onalies U, Schonenberger E, Krause E, O ksche A, Rosenthal W, Schulein R (2004) Disease-causing V (2) vasopressin receptors are retained in different compartments of the early secretory pathway. Traffic 5: 993-1005

Hu K, Huang CS, Jan YN, Jan LY (2003) ATP-sensitive potassium channel traffic regulation by adenosine and protein kinase $\mathrm{C}$. N euron $\mathbf{3 8}$ : 417-432

Khalil H, Brunet A, Saba I, Terra R, Sekaly RP, Thibodeau J (2003) The M HC class II $\beta$ chain cytoplasmic tail overcomes the invariant chain $p 35-$ encoded endoplasmic reticulum retention signal. Int Immunol 15: $1249-1263$

Kupershmidt S, Yang T, Chanthaphaychith S, Wang Z, Tow bin JA, Roden D M (2002) D efective human Ether-a-go-go-related gene trafficking linked to an endoplasmic reticulum retention signal in the $C$ terminus. J Biol Chem 277: $27442-27448$

Kuwana T, Peterson PA, Karlsson L (1998) Exit of major histocompatibility complex class II-invariant chain p35 complexes from the endoplasmic reticulum is modulated by phosphorylation. Proc N atl Acad Sci U SA 95: 1056-1061

Lee SK (1999) Four consecutive arginine residues at positions 836-839 of EBV gp110 determine intracellular localization of gp110. Virology 264: 350-358

Letourneur F, Hennecke S, D emolliere C, Cosson P (1995) Steric masking of a dilysine endoplasmic reticulum retention motif during assembly of the human high affinity receptor for immunoglobulin E. J Cell Biol 129: 971-978

Lotteau V, Teyton L, Peleraux A, Nilsson T, Karlsson L, Schmid SL, Q uaranta V, Peterson PA (1990) Intracellular transport of class II M HC molecules directed by invariant chain. $N$ ature 348: 600-605

M argeta-M itrovic M , Jan YN , Jan LY (2000) A trafficking checkpoint controls GABA (B) receptor heterodimerization. N euron 27: 97-106

M CM ahon HT, M ills IG (2004) CO P and clathrin-coated vesicle budding: different pathways, common approaches. Curr O pin Cell Biol 16: 379-391

M eyer GA, Radsak KD (2000) Identification of a novel signal sequence that targets transmembrane proteins to the nuclear envelope inner membrane. J Biol Chem 275: 3857-3866

M eyer G, Gicklhorn D, Strive T, Radsak K, Eickmann M (2002) A threeresidue signal confers localization of a reporter protein in the inner nuclear membrane. Biochem Biophys Res Commun 291: 966-971

Nilsson T, Jackson M, Peterson PA (1989) Short cytoplasmic sequences serve as retention signals for transmembrane proteins in the endoplasmic reticulum. Cell 58: 707-718

N ufer O, H auri HP (2003) ER export: call 14-3-3. Curr Biol 13: R391-R393

O 'Kelly I, Butler M H, Zilberberg N, Goldstein SA (2002) Forward transport. 14-3-3 binding overcomes retention in endoplasmic reticulum by dibasic signals. Cell 111: 577-588 
Ren Z, Riley NJ, Garcia EP, Sanders JM, Swanson GT, M arshall J (2003a) Multiple trafficking signals regulate kainate receptor KA2 subunit surface expression. J N eurosci 23: 6608-6616

Ren Z, Riley NJ, N eedleman LA, Sanders JM, Swanson GT, M arshall J (2003b) Cell surface expression of GluR5 kainate receptors is regulated by an endoplasmic reticulum retention signal. J Biol Chem 278: 52700-52709

Schutze M P, Peterson PA, Jackson M R (1994) An N-terminal double-arginine motif maintains type II membrane proteins in the endoplasmic reticulum. EMBO J 13: 1696-1705

Scott D B, Blanpied TA, Swanson GT, Zhang C, Ehlers M D (2001) An N M DA receptor ER retention signal regulated by phosphorylation and alternative splicing. J N eurosci 21: 3063-3072

Scott D B, Blanpied TA, Ehlers MD (2003) Coordinated PKA and PKC phosphorylation suppresses RXR-mediated ER retention and regulates the surface delivery of N M DA receptors. N europharmacology 45: 755-767

Shikano S, Li M (2003) M embrane receptor trafficking: evidence of proximal and distal zones conferred by two independent endoplasmic reticulum localization signals. Proc Natl Acad Sci U SA 100: 5783-5788

Standley S, Roche KW, M cCallum J, Sans N, Wenthold RJ (2000) PDZ domain suppression of an ER retention signal in N M DA receptor N R1 splice variants. N euron 28: 887-898

Teasdale RD, Jackson M R (1996) Signal-mediated sorting of membrane proteins between the endoplasmic reticulum and the Golgi apparatus. Annu Rev Cell Dev Biol 12: 27-54

Wang X, M atteson J, An Y, M oyer B, Yoo JS, Bannykh S, Wilson IA, Riordan JR, Balch WE (2004) CO PII-dependent export of cystic fibrosis transmembrane conductance regulator from the ER uses a di-acidic exit code. J Cell Biol 167: 65-74

Xia H, H ornby ZD, Malenka RC (2001) An ER retention signal explains differences in surface expression of N M DA and AM PA receptor subunits. Neuropharmacology 41: 714-723
Yuan H, Michelsen K, Schwappach B (2003) 14-3-3 dimers probe the assembly status of multimeric membrane proteins. Curr Biol 13: 638-646

Zerangue N, Schwappach B, Jan YN , Jan LY (1999) A new ER trafficking signal regulates the subunit stoichiometry of plasma membrane K(ATP) channels. Neuron 22: 537-548

Zerangue N, Malan MJ, Fried SR, D azin PF, Jan YN, Jan LY, Schwappach B (2001) Analysis of endoplasmic reticulum trafficking signals by combinatorial screening in mammalian cells. Proc $\mathrm{N}$ atl Acad Sci USA 98: 2431-2436

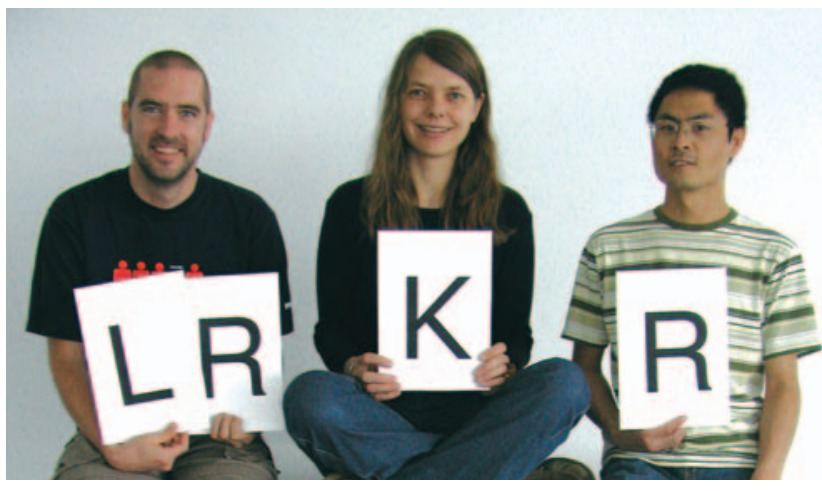

Kai Midhesen, BlandheSchwappachand Hebao Yuan. B.S. istheredieientof an EMBO Youngl nvestigator amerd. 